\title{
Interaction of microbiology and pathology in women undergoing investigations for infertility
}

\author{
Joseph Debattista ${ }^{1,2}$, Caroline M. Gazzard ${ }^{3}$, Robyn N. Wood ${ }^{3}$, John A. Allan ${ }^{4}$, \\ Janet M. Allan ${ }^{4}$, Anthony Scarman ${ }^{5}$, Miranda Mortlock ${ }^{6}$, Peter Timms ${ }^{2}$ and \\ Christine L. Knox ${ }^{2}$
${ }^{1}$ Sexual Health and AIDS Service, Prince Charles Hospital, Health Service District, Brisbane, Australia
${ }^{2}$ Centre for Molecular Biotechnology, Queensland University of Technology, Brisbane, Australia
${ }^{3}$ Queensland Medical Laboratory, West End, Brisbane, Australia
${ }^{4}$ Department of Reproductive Medicine, Wesley Hospital, Auchenflower, Brisbane, Australia
${ }^{6}$ School of Mathematics, Queensland University of Technology, Brisbane, Australia \\ ${ }^{5}$ Co-operative Research Centre, School of Life Sciences, Queensland University of Technology, Brisbane, Australia
}

Background: Cases of endometriosis with no tubal damage are associated with infertility, suggesting an immunological rather than mechanical barrier to reproduction. Laparoscopy and falloposcopy results of clinically asymptomatic women undergoing investigation of infertility were correlated with the outcomes of microbiological screening for Chlamydia trachomatis, Mycoplasma pneumoniae, Mycoplasma hominis, ureaplasma species, Neisseria gonorrhoeae, Neisseria meningitidis and Chlamydia pneumoniae.

Methods: A total of 44 women presenting to a hospital IVF service for laparoscopic or laparoscopic/ falloposcopic investigation of infertility provided endocervical swabs, fallopian tube washings, and peripheral whole blood for analysis.

Results: Of these 44 women, $15.9 \%$ (7) showed evidence of $C$. trachomatis infection as detected by either PCR or EIA serology. Of these 7 women, 5 (7I\%) had no or mild endometriosis and 2 (29\%) had moderate or severe endometriosis. Of the remaining 37 women who showed no evidence of chlamydial infection, 15 (40.5\%) had no or mild endometriosis.

Conclusion: Women with infertility, but without severe endometriosis at laparoscopy, showed a trend towards tubal damage and a higher rate of previous $C$. trachomatis infection. Although not statistically significant, this trend would suggest that, where moderate to severe tubal damage is found to be the primary cause of infertility, C. trachomatis infection could be a likely cause for such tubal damage.

Key words: CHLAMYDIA TRACHOMATIS; ENDOMETRIOSIS; FALlOPOSCOPY; TUBAL INFERTILITY;

UREAPLASMAS

\section{INTRODUCTION}

Intraluminal pathology of the fallopian tube is correlated with two conditions-pelvic inflammatory disease (PID) and endometriosis. The presence of such tubal disease is a strong determinant of infertility ${ }^{1} ; 20$ to $25 \%$ of infertile women have been shown to have endometriosis compared with 2 to $5 \%$ of fertile women ${ }^{2}$, and up to a third of women with endometriosis show evidence of salpingitis ${ }^{3}$. Whereas severe cases of endometriosis accompanied by extensive pelvic

Correspondence to: Joseph Debattista, Sexual Health \& AIDS Service, 270 Roma Street, Brisbane, QLD 4000, Australia.

Email: joedebat@powerup.com.au

(C) 2004 Parthenon Publishing. A member of the Taylor \& Francis Group Ltd.

DOI: 10.1080/I0647440400020703 
adhesions can lead to tubal infertility, mild or moderate cases with no tubal damage are also associated with infertility, suggesting an immunological rather than a mechanical barrier to reproduction $^{4,2}$.

PID also causes tubal infertility. The severity of tubal damage occurring during PID determines the probability of infertility: 3\% of women with mild tubal damage observed at laparoscopy, 13\% with moderate damage, and $29 \%$ with severe damage, were identified as having tubal infertility $^{5}$. Despite the high causal relationship between bacterial infection and PID, organisms are rarely isolated in PID cases, and even less frequently in cases of tubal infertility ${ }^{6}$.

Most women with tubal infertility do not have a history of recognised acute salpingitis. However, a number of previous studies investigating acute infections, pelvic inflammatory disease and salpingitis have demonstrated that Chlamydia trachomatis and Neisseria gonorrhoeae are the most common causes of such infection ${ }^{7-10}$; and others have demonstrated an abnormally high prevalence of antichlamydial (C. trachomatis) antibodies in women with tubal infertility and ectopic pregnancy ${ }^{11-15}$.

In this study we investigated the severity of intraluminal disease, endometriosis and evidence for microorganisms associated with PID among a cohort of clinically asymptomatic women undergoing laparoscopy or laparoscopy/falloposcopy for the investigation of infertility. The objective was to determine the prevalence of, and possible relationship between, asymptomatic infection and pathology of the fallopian tubes consequent to endometriosis and PID. This study reports on pathological findings at laparoscopy or laparoscopy/ falloposcopy, and outcomes of micro-biological screening for microorganisms including $C$. trachomatis, M. pneumoniae, $M$. hominis, ureaplasma species, N. gonorrhoeae and N. meningitidis, and serological testing for C. trachomatis, C. pneumoniae and M. pneumoniae.

\section{MATERIALS AND METHODS}

\section{Participants}

This study involved 44 women presenting to a hospital IVF service for laparoscopic and fallopo- scopic investigation of infertility. These women had a history of infertility of at least 2 years' duration, were clinically asymptomatic for PID, and either had a history of signs and symptoms suggestive of endometriosis, or a previous diagnosis of tubal disease as confirmed by previous laparoscopy, or longstanding unexplained infertility. All subjects had had other forms of infertility investigated (male factor, ovulation defects) before undergoing laparoscopy or laparoscopy/ falloposcopy. Informed consent was obtained from all participants and the study was approved by the human ethics committees of the Queensland University of Technology and the Wesley Hospital, Brisbane, Queensland.

\section{Clinical samples}

Three separate clinical samples were collected from each of the participants: endocervical swabs for culture and PCR analysis; fallopian tube washings for culture, PCR analysis and serology; and $10 \mathrm{ml}$ peripheral whole blood for serological assay. The endocervical swabs were collected during a speculum examination before the laparoscopy/falloposcopy procedure. The cervix was then prepared for falloposcopy and swabbed with povidone iodine. As a matter of protocol, all women were treated with antibiotics before the insertion of instrumentation through the cervix, in order to reduce the possibility of pelvic infection due to contamination carried from the vagina. Cefotaxamine sodium, $2 \mathrm{~g}$ intravenously, was administered at the commencement of the procedure.

During falloposcopy an endoscope (Imagyn Medical, Switzerland) was inserted through the cervix and uterus into the fallopian tubes. The tubes were visualised along their length by means of a linear everting catheter and falloposcope. Saline was passed through the fallopian tube and any tissue debris or adhesions within the tubal lumen were cleared if possible during this procedure. The resultant cellular debris and saline were collected from the pouch of Douglas by aseptic aspiration via laparoscopy and aliquotted for testing. In 5 cases severe tubal pathology precluded falloposcopic examination, although saline was forced through under pressure. Thus 
fallopian tube washings were collected in these 5 cases although falloposcopic observation was not possible.

\section{Classification of endometriosis}

The classification of endometriosis employed in this study was a modification of the American system, developed specifically with reference to fertility outcomes. Laparoscopic observations of endometriosis were classified according to the extent and distribution of endometriosis and the presence of adhesions. The presence of some peritoneal endometriosis deposits, with no endometriosis on tubes or ovaries and no pelvic adhesions, was classed as mild. The presence of peritoneal endometriosis deposits and/or pelvic adhesions, with superficial endometriosis deposits on the ovarian capsule but no periovarian or peritubal adhesions, was classed as moderate. Endometrioma formation in the ovaries, extensive peritoneal endometriosis, extensive pelvic adhesions, and deposits of endometriosis on tubal serosa with or without peritubal/periovarian adhesions constituted severe endometriosis.

\section{Classification of intraluminal disease}

Falloposcopy findings observed inside the fallopian tubes were classified as follows. Minimal loss of intraluminal folds with no adhesions or obstruction in either fallopian tube was classed as mild. Complete obstruction of one or incomplete obstruction of either one or both tubes, with moderate adhesions and moderate loss of tubal mucosal folds, was classed as moderate. Intraluminal tubal pathology (found at falloposcopy to be the primary cause of infertility) with permanent bilateral obstruction or extensive loss of normal intraluminal tubal architecture (presence of intratubal adhesions and loss of tubal mucosal folds) constituted severe disease.

\section{Detection of $C$. trachomatis and neisseria species.}

Endocervical swabs were tested by Amplicor CT/NG PCR (Roche Diagnostics, Branchburg, NJ, USA) for both $C$. trachomatis and $N$. gonorrhoeae (according to the manufacturer's instructions), and cultured for N. meningitidis using chocolate agar incubated overnight at $35^{\circ} \mathrm{C}$. Samples of fallopian tube washings obtained from falloposcopy and/or laparoscopy were also tested for C. trachomatis by the Roche Amplicor CT/NG PCR and cultured for both $N$. gonorrhoeae and N. meningitidis using Thayer Martin/chocolate agar. The Roche Amplicor $\mathrm{CT} / \mathrm{NG}$ PCR method incorporates an internal control to monitor for the presence of PCR inhibitors.

\section{Antibody screening}

Peripheral blood serum and fallopian tube washings collected from participants were tested for anti-C. trachomatis IgG and IgA antibodies and anti-C. pneumoniae $\operatorname{IgG}$ antibodies. $C$. trachomatis antibodies were detected using the Labsystems Chlamydia trachomatis IgG EIA and IgA EIA (Helsinki, Finland). C. pneumoniae IgG antibodies were detected by immunofluorescence using the FOCUS Chlamydia MIF (Los Angeles, USA). An agglutination assay (Fujirebio Serodia-MYCOII, Japan) was selected for determination of $M$. pneumoniae antibodies using washings only.

Serum samples were tested and interpreted for C. trachomatis and C. pneumoniae antibodies according to the manufacturer's instructions. As no criteria were supplied for samples other than blood, the following adaptations were made for testing the fallopian fluids. Samples of $C$. trachomatis were initially tested undiluted; if positive, they were retested at dilutions of 1:2 and 1:10 prepared using sample diluent. C. pneumoniae samples were initially tested at 1:16 dilution; and $M$. pneumoniae samples were initially tested at 1:4 dilution.

\section{Ureaplasma/ mycoplasma detection}

\section{Ureaplasma culture}

Endocervical swabs (39 samples tested) and fallopian tube washings (40 samples tested) were inoculated directly into $10 \mathrm{~B}$ broth $^{16}$ and then held at $-80^{\circ} \mathrm{C}$ until testing. In the laboratory, 
four serial 10-fold dilutions were made of the liquid broth, which was then incubated at $37^{\circ} \mathrm{C}$ until ureaplasma growth was detected by an alkaline shift or for 3 weeks.

\section{Ureaplasma/mycoplasma PCR detection}

All clinical samples were prepared for extraction of DNA by the method of Blanchard et al ${ }^{17}$. All endocervical and fallopian tube clinical samples were tested by PCR assay for the presence of ureaplasmas. M. hominis and all endocervical ureaplasma isolates identified as $U$. parvum were further subtyped using four separate single-step mba PCR assays by previously described methods $^{18}$. For M. pneumoniae testing, primers specific for the species were designed using the P1 and ORF453 genes of $M$. pneumoniae and an annealing temperature of $52^{\circ} \mathrm{C}$. Primers were designed to avoid regions of high homology with $\mathrm{Myco-}$ plasma genitalium and Ureaplasma urealyticum sequences. ORF 453 encodes a possible protoporphyrinogen oxidase, and primers ORF-F (5' TCAACTACCCT-TACCCTATTACC 3') and ORF-R (5' GCACTCCAGTATCACTTGTTTAAC 3') generate an amplicon of $505 \mathrm{bp}$. Primers to the P1 major adhesin of $M$. pneumoniae were designed in the constant region 1 to avoid repetitive elements ${ }^{19}$ and variable regions ${ }^{20}$. PCR amplification using primers $\mathrm{P} 1-\mathrm{F} \quad\left(5^{\prime}\right.$ CTTACTGT-TCTCAGGCATGG 3') and P1R (5' TTGACAAGACCGTCCA-ATCC 3') produced a fragment of $383 \mathrm{bp}$. M. pneumoniae DNA kindly supplied by G. Browning, University of Melbourne, was used as a control. The PCR reaction mixture and thermal cycling conditions were those previously described ${ }^{18}$.

\section{Other bacteriological culture}

An endocervical swab was also cultured on horse blood and Sabouraud's dextrose agar and incubated overnight at $35^{\circ} \mathrm{C}$.

\section{Statistical analysis}

Associations of chlamydia infection between women with nil/mild and moderate/severe endometriosis and tubal disease were examined by two-way tables using chi-squared and Fisher's exact tests.

\section{RESULTS}

\section{Laparoscopy and falloposcopy results}

Of the 44 women enlisted in this study, 5 underwent a laparoscopy procedure but did not proceed to falloposcopy; however, in these cases fallopian tube washings were obtained as a result of saline being washed through. All of these 5 women were noted to have peritubal adhesions on external examination by laparoscopy, and their tubal disease was consequently classified as moderate to severe. On laparoscopy, 40 (90.9\%) of the 44 women were found to have endometriosis which was classified as mild in 16 (36.4\%) cases, as moderate in $15(34.1 \%)$ cases, and as severe in $9(20.5 \%)$ cases. The presence of fallopian tubal disease as identified by falloposcopy and/or external laparoscopy was identified as moderate to severe in 38/44 (86.4\%) participants, and nil or mild in the remaining 6 (13.6\%) participants. Of the other 4 women who recorded no diagnosis of endometriosis, all recorded severe tubal obstructions on falloposcopy; 7 women with mild endometriosis also showed severe, bilateral obstructions within the fallopian tubes.

\section{Endocervical culture/PCR results}

Ureaplasmas were detected by culture and PCR in $11 / 39(28.2 \%)$ of the endocervical samples tested and were identified as $U$. parvum. These $U$. parvum isolates were subsequently subtyped; 7/12 (58\%) were serovar 3 isolates, 1/12 (8.3\%) were serovar 1 , and 4/12 (33\%) were serovar 6. Mixed serovars 1 and 3 were detected in 1 of the endocervical samples. M. hominis, M. pneumoniae, C. trachomatis, $N$. gonorrhoeae and $N$. meningitidis were not detected in any of the endocervical samples by PCR and/or culture. Culture of endocervical samples further identified mild growth of bacteroides species in $1 / 44(2.3 \%)$ women, mild growth of Candida albicans in 1/44 women (2.3\%), and moderate growth of group B streptococcus in 5/44 (11.4\%) women, 1 of whom was coinfected with Gardnerella vaginalis. 
Endocervical cultures for the remaining women grew the bacterial flora that could normally be expected after vaginal contamination.

\section{Fallopian tube culture/PCR results}

C. trachomatis was detected in the fallopian tubes of 1 woman (Table 1). Serology for both fallopian washings and serum for this woman was found to be negative for $C$. trachomatis, and no other fallopian tube sample was found to be positive for C. trachomatis. Ureaplasmas, M. hominis, $M$. pneumoniae, N. gonorrhoeae and N. meningitidis were not detected in any of the fallopian tube samples.

\section{Antibody testing}

Both serum and fallopian tube fluids were available for serological testing for 38 of the 44 women; for 3 women only serum was tested and for 3 only fallopian tube fluid was tested.

\section{Peripheral blood serology}

Of the 41 serum samples, 4 (9.8\%) were positive for C. trachomatis $\mathrm{IgG}$, and 1 (2.4\%) for C. trachomatis IgA (Table 1). Positive results for both $\operatorname{IgG}$ and IgA anti-C. trachomatis antibodies were found in 1 sample, and 1 demonstrated a positive $\operatorname{IgG}$ and an equivocal $\operatorname{IgA}$ anti-C. trachomatis antibody level; 25/36 samples (69.4\%) were positive for C. pneumoniae IgG.

\section{Fallopian tube serology}

Of the 41 fallopian tube washings, 5 (12.2\%) were positive for the presence of $C$. trachomatis $\operatorname{IgG}, 1$ (2.4\%) for C. trachomatis IgA (Table 1), 5 (12.2\%) for C. pneumoniae $\operatorname{IgG}$, and 1 (2.4\%) for $M$. pneumoniae serology. In 1 case both $\operatorname{IgG}$ and $\operatorname{IgA}$ anti-C. trachomatis antibodies were found in both the fallopian tube and serum.

\section{Association of laparoscopic and falloposcopic findings with serology/microbiological results}

Evidence (either in peripheral blood or fallopian tube washings) of $C$. trachomatis infection was found in 7 women (Table 1). Nil or mild endometriosis was diagnosed in 5 (71\%) of these, and 4 of the 5 were also shown to have moderate to severe intraluminal disease according to falloposcopic observation. Participant 32 (Table 1) showed no evidence of endometriosis but had severe tubal disease with extensive bilateral damage. Only participants 14 and 31 (Table 1) had endometriosis classified as moderate and severe, respectively, and both these women had severe tubal disease showing extensive adhesions and scarring. Participant 14 was also the 1 woman with a $C$. trachomatis infection in the fallopian tube detectable by PCR, although serology was negative. Therefore, in total, $6(86 \%)$ of the 7 women with evidence of chlamydial infection showed moderate to severe intraluminal disease

Table I Combined results of serum and fallopian tube C. trachomatis pathology

\begin{tabular}{|c|c|c|c|c|c|c|}
\hline Case & Serum IgG & Serum $\lg A$ & Fallopian $\lg G$ & Fallopian $\lg A$ & Endometriosis & $\begin{array}{c}\text { Intraluminal } \\
\text { disease }\end{array}$ \\
\hline Patient 12 & + & - & + & - & Mild & Moderate \\
\hline $\begin{array}{l}\text { Patient } 14, \text { PCR } \\
+ \text { ve }\end{array}$ & - & - & - & - & Moderate & Severe \\
\hline Patient 17 & + & Equivocal & - & - & Mild & Severe \\
\hline Patient 29 & + & - & + & - & Mild & Nil \\
\hline Patient 31 & - & - & + & - & Severe & Severe \\
\hline Patient 32 & + & + & + & + & Nil & Severe \\
\hline Patient 36 & - & - & + & - & Mild & Severe \\
\hline Total positives & 4 & 1 & 5 & I & & \\
\hline Total patients & 41 & 41 & 41 & 41 & & \\
\hline $\begin{array}{l}\text { Percentage } \\
+ \text { ve }\end{array}$ & $9.8 \%$ & $2.4 \%$ & $12.2 \%$ & $2.4 \%$ & & \\
\hline
\end{tabular}


but only $2(28.6 \%)$ of these 7 women had moderate to severe endometriosis.

In 1 case with positive mycoplasma serology (fallopian tube fluid), mild endometriosis was defined, with severe tubal disease and a history of ruptured appendicitis.

Of the remaining 37 women who showed no microbiological evidence of chlamydial infection, $11(29.7 \%)$ had a diagnosis of mild endometriosis, $13(35.1 \%)$ of moderate endometriosis, and 9 $(24.3 \%)$ of severe endometriosis, whereas 4 (10.8\%) had no endometriosis. According to falloposcopic observations, 5 (13.5\%) of women in this same group had no or mild tubal disease and $32(86.5 \%)$ had moderate to severe tubal disease.

The laparoscopy and falloposcopy results for those women with $C$. trachomatis positivity, and those with none, were grouped into two general categories of endometriosis and tubal disease (nil/ mild and moderate/severe) and are described in Table 2.

Among the women with evidence of chlamydial infection, $71 \%$ had a diagnosis of nil $/$ mild endometriosis diagnosis compared with $40.5 \%$ of women with no evidence of chlamydia $(p>0.5)$. Among the women with evidence of chlamydia and classifications of nil/mild endometriosis, $56.7 \%$ showed moderate/severe tubal damage compared with $32.4 \%$ of women without positive chlamydia serology and with nil/mild endometriosis $(p>0.5)$.

The 9 women with endocervical ureaplasma infection were evenly distributed across both

Table 2 C. trachomatis pathology in different categories of endometriosis and intraluminal disease: numbers (\%) of cases

\begin{tabular}{lcc}
\hline & $\begin{array}{c}\text { Nil/mild } \\
\text { endometriosis }\end{array}$ & $\begin{array}{c}\text { Moderate/ } \\
\text { severe } \\
\text { endometriosis }\end{array}$ \\
\hline Pubal disease & & \\
Positive for C. trachomatis & $\mathrm{I}(14.3 \%)$ & - \\
-nil/mild & $4(56.7 \%)$ & $2(29.0 \%)$ \\
-moderate/severe & $5(71.0 \%)$ & $2(29.0 \%)$ \\
-total $(n=7)$ & $3(8.1 \%)$ & $2(5.5 \%)$ \\
-negative for C. trachomatis & $12(32.4 \%)$ & $20(54.0 \%)$ \\
-moderate/severe & $15(40.5 \%)$ & $22(59.5 \%)$ \\
-total $(n=37)$ & & \\
\hline
\end{tabular}

groups of endometriosis: $44 \%$ had nil/mild endometriosis and 56\% had moderate/severe endometriosis.

A review of the clinical histories of the participants showed that of the 7 women with evidence of $C$. trachomatis infection, 1 gave a history of miscarriage, 1 gave a history of ectopic pregnancy, and 2 had had PID previously. Another 2 participants in this group displayed significant tubal disease indicative of previous infection. In comparison, the clinical histories of the other 37 women included (in addition to endometriosis) 5 cases of polycystic ovarian disease, 4 of irregularities with ovulation, 1 of possible PID at a very early age (no tubal disease was detected), 1 of hydrosalpinx, 1 of suspected infection from a previous termination of pregnancy and 2 of appendicitis.

When serum samples were assayed for $C$. pneumoniae, equivalent proportions of patients with either nil/mild or moderate/severe endometriosis were found to have positive serology for C. pneumoniae (44\% and 56\%, respectively).

\section{DISCUSSION}

C. trachomatis is now recognised as one of the most common sexually transmissible bacterial pathogens throughout the world. C. trachomatis has previously been found to be the primary cause of salpingitis and pelvic inflammatory disease (30 to $60 \%$ of cases $)^{7-10,21}$, often with consequent ectopic pregnancy ${ }^{22}$ and tubal infertility ${ }^{23,24}$. The presence of $C$. trachomatis DNA at sites of inflammation and tissue damage within reproductive tissue in both animal models ${ }^{25}$ and humans with PID and tubal infertility ${ }^{26-29}$ suggests that chlamydia persist within the fallopian tube in a non-replicative chronic state and may be directly involved in the stimulation of the immune-mediated tissue destruction.

However, despite strong evidence of $C$. trachomatis involvement in tubal infertility, isolation of the organism by culture is $\operatorname{rare}^{28}$. Falloposcopy as performed for this cohort of women remains one of the few options for obtaining fallopian tissue for analyses. However, in our study tissue biopsies were not taken. In view of the intracellular reproductive cycle of $C$. 
trachomatis, the collection of luminal tissue for testing is ideal, but the process of clearing out debris by means of the passage of saline through the fallopian tube was considered sufficient in our study to provide suitable material for PCR testing. Nonetheless, one deficiency in this approach was our failure to collect pre-existing cul-de-sac fluid before aspiration of the tubal effluent.

This study reports the microbiological screening of cervical, serum and fallopian tube samples collected from women undergoing laporoscopic and/or falloposcopic investigation for infertility. A total of 7 women (15.9\%) within our cohort of 44 women showed evidence of previous $C$. trachomatis infection, either in the form of serum $\operatorname{IgG}(n=4)$ and/or IgA antibodies $(n=1)$, fallopian tube $\operatorname{IgG}(n=5)$ and/or $\operatorname{IgA}(n=1)$ antibodies, or chlamydial DNA detected by PCR in the fallopian tube washings $(n=1)$. This prevalence, recorded in a population of women accessing an infertility service, is as expected higher than both the general and antenatal prevalence rates. The prevalence of 3 to $5 \%$ in the general community was calculated from urine-based PCR among, for example, adult clients attending the Brisbane Sexual Health Clinic on any given month (unpublished data). The antenatal prevalence of $3.7 \%$ was previously reported in antenatal patients attending a public hospital clinic $^{30}$.

The prevalence (15.9\%) found in this study is comparable with that reported by other studies. In a previous study of 1303 subfertile couples, asymptomatic for genital tract infection, elevated titres of IgG anti-chlamydial antibodies were found in $20.8 \%$ of all women ${ }^{11}$. Similarly Vogelzang et al. ${ }^{31}$, in a study of infertile women referred to an IVF provider for diagnostic laparoscopy, found that $7 / 33(21.2 \%)$ women with tubal disorders (adhesions, distal occlusions and strictures) demonstrated antibodies to $C$. trachomatis compared with $0 / 20$ of women without tubal damage. Further studies have also strongly supported the link between serological evidence of previous C. trachomatis infections and tubal infertility and ectopic pregnancies ${ }^{12-15}$.

The detection of antibodies to $C$ trachomatis within fallopian tube washings collected does not necessarily reflect actual antibody production in the tubes but could also be the result of serum transudate or contamination by blood washed through with the dislodged cellular debris and fragments of endometrium.

In this current study we also compared the prevalence and severity of endometriosis in women positive and negative for $C$. trachomatis. Although there was no statistical significance, there appeared to be a trend in which a higher proportion of those infertility clinic clients returning a positive $C$. trachomatis diagnosis recorded nil/mild categories of endometriosis (5/7: 71\%) compared with those women without any evidence for $C$. trachomatis (15/37: 40.5\%). Also, there was a trend in which a lower proportion of positive $C$. trachomatis diagnoses recorded moderate/severe categories of endometriosis $(2 / 7: 29 \%)$ compared with the negative group (22/37: 59.5\%). Again this was not significant in view of the small numbers. These results suggest a negative relationship between $C$. trachomatis and endometriosis. Therefore, in this current study, endometriosis appears not to be associated with a previous history of $C$. trachomatis infection. A previous study had similarly found that only $1 / 15$ patients with endometriosis had detectable antibodies to $C$. trachomatis ${ }^{14}$.

The majority of participants in this present study were found during falloposcopy to have moderate/severe intraluminal disease (38/44: 86.4\%). Moderate/severe tubal damage was observed in women with both nil/mild and moderate/severe endometriosis $(87.1 \%$ and $80.4 \%$, respectively). Therefore the severity of the tubal damage did not relate to the endometriosis classification. 16/44 (36\%) of the women in our study presented with moderate to severe tubal damage in the absence of moderate to severe endometriosis. Although 25\% (4/16) of these women showed evidence of $C$. trachomatis infection, the aetiology of tubal damage for the remaining 12 women remained undefined.

When both endometriosis and tubal disease were investigated together, $56.7 \%$ of participants with positive $C$. trachomatis findings had moderate/severe tubal disease and nil/mild endometriosis, whereas only $32.4 \%$ of participants without evidence of $C$. trachomatis infection had 
similar categories of intraluminal disease and endometriosis. Therefore the findings of tubal damage in those women with evidence of $C$. trachomatis may be due to this previous infection, in view of the fact that endometriosis was predominantly mild or non-existent for this group.

A more speculative explanation for the higher prevalence of $C$. trachomatis among this small sample of women with nil/mild endometriosis may be a protective effect. Could C. trachomatis infection limit the extent and severity of endometriosis or, conversely, does a pre-existing endometriosis condition limit C. trachomatis infection? It may be possible that tubal occlusion resulting from C. trachomatis infection may create a physical barrier against endometrial tissue passing through the fallopian tube or, alternatively, immune responses generated during endometriosis such as elevated cytokine expres$\operatorname{sion}^{2,32,33}$, increased neutrophil activity ${ }^{34-36}$ and increased phagocytic activity ${ }^{37,38}$ may clear chlamydial colonisation before the development of florid infection.

The prevalence of C. pneumoniae antibodies in sera was evenly distributed across both classifications of endometriosis (Table 3), and there did not appear to be an association with $C$. trachomatis serology. Freidank et al. ${ }^{39}$ showed a higher anti-hsp60 antibody rate in patients with complete tubal occlusion (76\%) than in infertility patients with normal fallopian tubes (19\%), and this was associated with the presence of antibodies not only to $C$. trachomatis but also to C. pneumoniae, suggesting a synergistic effect due to cross-reactivity. However, Osser and Persson $^{40}$ had earlier argued against the possibility of cross-reactivity due to shared antigens by demonstrating that antibodies to $C$. pneumoniae did not influence the frequency or titre of

Table 3 Correlation of C. pneumoniae serology with endometriosis rating: numbers (\%) of cases

\begin{tabular}{lcc}
\hline Endometriosis & $\begin{array}{c}\text { Positive C. } \\
\text { pneumoniae } \\
\text { rating }\end{array}$ & $\begin{array}{c}\text { Negative C. } \\
\text { pneumoniae } \\
\text { serology }(n=25)\end{array}$ \\
\hline Nil/mild & $11(44 \%)$ & $6(55 \%)$ \\
Moderate/severe & $14(56 \%)$ & $5(45 \%)$ \\
\hline
\end{tabular}

antibodies to C. trachomatis in patients recovering from acute salpingitis. C. trachomatis antibodies, however, did reflect the severity of inflammatory involvement. More recently ${ }^{41}$, patients with tubal factor infertility were shown to have significantly higher frequencies and titres for all antibodies (C. trachomatis hsp60, hsp10, common chlamydial lipopolysaccharide) except to $C$. pneumoniae. No interaction between C. trachomatis and $C$. pneumoniae suggestive of a synergistic effect was found. The results of our study would suggest a similar trend, although the numbers of patients were too small for statistical significance.

In this study we also tested endocervical swabs and fallopian tube washings for the presence of the ureaplasmas (U. parvum, $U$. urealyticum), $M$. hominis and $M$. pneumoniae. Whereas M. hominis and M. pneumoniae were not detected in either endocervical or fallopian tube samples, $28 \%$ of the women tested were found to have $U$. parvum in the endocervix. However, no ureaplasmas were detected in the fallopian tube washings by either culture or PCR. Testing of the fallopian tube specimens using a more sensitive nested $m b a$ PCR assay (results not shown) also failed to detect ureaplasmas within these specimens. These results confirm that the fallopian tube specimens had not been contaminated during the transcervical falloposcopy procedure.

Previous studies demonstrated a very low isolation rate of the ureaplasmas (0 to $3 \%$ ) and M. hominis (0 to $4 \%$ ) from the fallopian tubes ${ }^{7,9}$. This is despite higher ureaplasma/M. hominis colonisation rates in the endocervix (48 to 52\%) and the endometrium (6 to 30\%). However, these studies highlighted the increased prevalence of $C$. trachomatis: $30 \%$ of women were infected with $C$. trachomatis in the endometrium and 17 to $22 \%$ of women demonstrated infection within fallopian tubes $^{7,9}$. M. pneumoniae was not detected in either endocervical or fallopian tube samples in this current study, but this microorganism has been associated with orogenital contact and has been isolated from cervical and male urethral swabs ${ }^{42}$. A rare case of tubo-ovarian abscess has also been reported after pneumonia, indicating the possibility of systemic spread ${ }^{43}$. 
Our study also shows that there is no association between the prevalence of endometriosis and $U$. parvum, given that $44.4 \%$ of women with endocervical ureaplasmas had nil/mild endometriosis compared with $55.6 \%$ of ureaplasmapositive women with moderate/severe endometriosis.

N. meningitidis, another respiratory pathogen and coloniser, has also been isolated from the female genitourinary tract in both symptomatic and asymptomatic states, and is generally associated with orogenital contact ${ }^{43}$. Symptomatic infections range from urethritis to purulent cervicitis and salpingitis. In our population sample of 44 women, no N. meningitidis was isolated from either cervix or fallopian tube by culture. Whereas the prevalence of anogenital infection in the UK has remained very low at $0.13 \%$, other studies in the USA have isolated N. meningitidis in $20 \%$ of cases urogenital detection of pathogenic Neisseria species ${ }^{43}$.

Other organisms identified in the endocervix of these women were considered consistent with lower genital tract flora, as no participants in the study had symptoms of PID or vaginitis.

The evidence for $C$. trachomatis infection identified in $15.9 \%$ of women involved in this study was comparable with previous studies and was certainly higher than the prevalence (3.7\%) within a population of fertile Australian wo$m^{3}{ }^{30}$. The absence of $C$. trachomatis indicators in women with moderate to severe endometriosis, and the comparable rates of women with and without evidence for ureaplasma across all groupings of endometriosis, supports a non-microbial cause for endometriosis. Further, this study may suggest a protective effect by which previous chlamydial-induced tubal damage may limit the spread of endometriosis, or immune responses associated with endometriosis may prevent chlamydia infection. Our study also suggested that there was no synergistic link between $C$. pneumoniae and $C$. trachomatis infection with respect to inducing tubal damage. Finally, a third of the women in our group who presented with moderate to severe intraluminal disease had no significant evidence of endometriosis. Whereas $25 \%$ of this group showed some evidence for previous C. trachomatis infection, there remained a majority with no apparent microbiological cause for their tubal disease.

\section{Acknowledgements}

This project was funded by the Wesley Research Institute. The project received the support of consumables from Sullivan and Nicolaides Pathology, Queensland Medical Laboratory and the Division of Microbiology, Royal Brisbane Hospital. We also thank the laboratory staff of the Division of Microbiology, Royal Brisbane Hospital for performing the specimen culture and PCR, and G. Browning for the M. pneumoniae DNA.

\section{REFERENCES}

1. Bowman MC, Cooke ID. Comparison of fallopian tube intraluminal pathology as assessed by salpingoscopy with pelvic adhesions. Fertil Steril 1994;61:464-9

2. Harada $T$, Iwabe $T$, Terakawa N. Role of cytokines in endometriosis. Fertil Steril 2001;76: $1-10$

3. Forrest J, Buckley HJ, Fox H. Pelvic endometriosis and tubal inflammatory disease. Int $J$ Gynaecol Pathol 1984;3:343-7

4. Koyama N, Matsuura K, Okamura H. Cytokines in the peritoneal fluid of patients with endometriosis. Int J Gynecol Obstet 1993;43:45-50
5. Pavletic AJ, Wolner-Hanssen P, Paavonen J, et al. Infertility following pelvic inflammatory disease. Infect Dis Obstet Gynaecol 1999;7:145-52

6. Guerra-Infante FM, Flores-Medina S, LopezHurtado $M$, et al. Tumor necrosis factor in peritoneal fluid from asymptomatic infertile women. Arch Med Res 1999;30:138-3

7. Heinonen PK, Miettinen A. Laparoscopic study on the microbiology and severity of acute pelvic inflammatory disease. Eur J Obstet Gynecol Reprod Biol 1994;57:85-9 
8. Miettinen A. Mycoplasma hominis in patients with pelvic inflammatory disease. Isr J Med Sci 1987;23: 713-6

9. Stacey CM, Munday PE, Taylor-Robinson D, et al. A longitudinal study of pelvic inflammatory disease. Br J Obstet Gynaecol 1992;99:994-9

10. Svensson L, Westrom L, Mardh PA. Acute salpingitis with Chlamydia trachomatis isolated from the fallopian tubes: clinical, cultural, and serologic findings. Sex Transm Dis 1981;8:51-5

11. Eggert-Kruse W, Rohr G, Demirakca T, et al. Chlamydial serology in 1303 asymptomatic subfertile couples. Hum Reprod 1997;12:1464-75

12. Henry-Suchet J, Utzmann C, De Brux J, et al. Microbiologic study of chronic inflammation associated with tubal infertility: role of Chlamydia trachomatis. Fertil Steril 1987;47:274-7

13. Keay SD, Barlow R, Eley A, et al. The relation between immunoglobulin $\mathrm{G}$ antibodies to Chlamydia trachomatis and poor ovarian response to gonadotrophin stimulation before in vitro fertilisation. Fertil Steril 1998;70:214-18

14. Moore DE, Spadoni LR, Foy HM, et al. Increased frequency of serum antibodies to Chlamydia trachomatis in infertility due to distal tubal disease. Lancet 1982;574-7

15. Shepard MK, Jones RB. Recovery of Chlamydia trachomatis from endometrial and fallopian tube biopsies in women with infertility of tubal origin. Fertil Steril 1989;52:232-8

16. Shepard MC, Lunceford CD. Serological typing of Ureaplasma urealyticum isolates from urethritis patients by an agar growth inhibition method. $J$ Clin Microbiol 1978;8:566-74

17. Blanchard A, Gautier M, Mayau V. Detection and identification of mycoplasmas by amplification of rDNA. FEMS Microbiol Letters 1991;81:37-42

18. Knox CL, Allan JA, Allan JM, et al. Ureaplasma parvum and Ureaplasma urealyticum detected in semen after washing prior to assisted reproductive technology procedures. Fertil Steril, in press

19. Ruland K, Wenzel R, Herrmann R. Analysis of three different repeated DNA elements present in the P1 operon of Mycoplasma pneumoniae: size, number and distribution on the genome. Nucleic Acids Res 1991;11:6311-7

20. Kenri T, Taniguchi R, Sasaki Y, et al. Identification of a new variable sequence in the $\mathrm{P} 1$ cytadhesin gene of Mycoplasma pneumoniae: evidence for the generation of antigenic variation by DNA recombination between repetitive sequences. Infect Immun 1999; 67:4557-62
21. Westrom L, Mardh PA. Acute pelvic inflammatory disease. In: K.K. Holmes, P-A. Mardh, P.F. Sparling, P.J. Wiesner, editors. Sexually transmitted diseases. New York: McGraw-Hill; 1990. p 593-615

22. Wolner-Hanssen P, Kiviat NB, Holmes KK. Atypical pelvic inflammatory disease: subacute, chronic, or subclinical upper genital tract infection in women. In: K.K. Holmes, P-A. Mardh, P.F. Sparling, P.J. Wiesner, editors. Sexually transmitted diseases. New York: McGraw-Hill; 1990. p 615-21

23. Moore DE, Cates W. Sexually transmitted diseases and infertility. In: K.K. Holmes, P-A. Mardh, P.F. Sparling, P.J. Wiesner, editors. Sexually transmitted diseases. New York: McGraw-Hill; 1990. p 763-71

24. Paavonen J. Pelvic inflammatory disease. Sex Transm Dis 1998;16:747-55

25. Cappuccio AL, Patton DL, Kuo CC, Campbell LA. Detection of Chlamydia trachomatis deoxyribonucleic acid in monkey models (Macaca nemestrina) of salpingitis by in situ hybridization: implications for pathogenesis. Am J Obstet Gynecol 1994;171:102-10

26. Barlow REL, Cooke ID, Odukoya O, et al. The prevalence of Chlamydia trachomatis in fresh tissue specimens from patients with ectopic pregnancy or tubal factor infertility as determined by PCR and in-situ hybridisation. J Med Microbiol 2001; 50:902-8

27. Campbell LA, Patton DL, Moore DE, et al. Detection of Chlamydia trachomatis deoxyribonucleic acid in women with tubal infertility. Fertil Steril 1993;59:45-50

28. Hinton EL, Bobo LD, Wu TC, et al. Detection of Chlamydia trachomatis DNA in archival paraffinized specimens from chronic salpingitis cases using the polymerase chain reaction. Fertil Steril 2000;74: 152-7

29. Toth M, Patton DL, Campbell LA, et al. Detection of chlamydial antigenic material in ovarian, prostatic, ectopic pregnancy and semen samples of culture-negative subjects. Am J Reprod Immunol 2000;43:218-22

30. Knox CL, Cave DG, Farrell DJ, et al. The role of Ureaplasma urealyticum in adverse pregnancy outcome. Aust NZ J Obstet Gynaecol 1997;37:45-51

31. Vogelzang AA, van Joost T, Stolz E, Michel MF. Prevalence of antibodies to Chlamydia trachomatis, Neisseria gonorrhoeae, and Mycoplasma hominis in infertile women. Genit Med 1985;61:175-8 
32. Fang X, Lin Q, Chen Y. Study on in-vitro cytokines levels induced from peripheral mononuclear cells in patients with endometriosis. Zhonghua Fu Chan Ke Za Zhi 1999;34:609-11

33. Iwabe $\mathrm{T}$, Harada $\mathrm{T}$, Terakawa $\mathrm{N}$. Role of cytokines in endometriosis-associated infertility. Gynecol Obstet Invest 2002;53:19-25

34. Arici A. Local cytokines in endometrial tissue: the role of interleukin-8 in the pathogenesis of endometriosis. Ann NY Acad Sci 2002;955:101-9

35. Kamer-Bartosinska A, Szyllo K, Tchorzewski H, et al. Evaluation of peripheral blood neutrophils activity in women with endometriosis. Ginekol Pol 2001;72:431-6

36. Kwak JY, Park SW, Kim KH, et al. Modulation of neutrophil apoptosis by plasma and peritoneal fluid from patients with advanced endometriosis. Hum Reprod 2002;17:595-600

37. Gogacz M, Jakowicki J, Kotarski J. Phagocyte activity of macrophages in the peritoneal fluid of patients with endometriosis. Ginekol Pol 2001;72: $402-7$

38. Osborn BH, Haney AF, Misukonis MA, Weinberg JB. Inducible nitric oxide synthase expression by peritoneal macrophages in endometriosisassociated infertility. Fertil Steril 2002;77:46-51
39. Freidank HM, Clad A, Herr AS, et al. Immune response to Chlamydia trachomatis heat-shock protein in infertile female patients and influence of C. pneumoniae antibodies. Eur J Clin Micro Infect Dis 1995;14:1063-9

40. Osser S, Persson K. Immune response to genital chlamydial infection and influence of C. pneumoniae (TWAR) antibodies. Euro J Clin Microbiol Infect Dis 1989;8:532-5

41. Persson K, Osser S, Birkelund S, et al. Antibodies to Chlamydia trachomatis heat shock proteins in women with tubal factor infertility are associated with prior infection by $C$. trachomatis but not by C. pneumoniae. Human Reprod 1999;14:1969-73

42. Goulet M, Dular R, Tully JG, et al. Isolation of Mycoplasma pneumoniae from the human urogenital tract. J Clin Microbiol 1995;33:2823-2825

43. Edwards S, Carne C. Oral sex and the transmission of non-viral STIs. Sex Transm Infect 1998;74: 95-100 


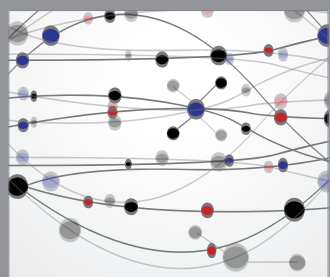

The Scientific World Journal
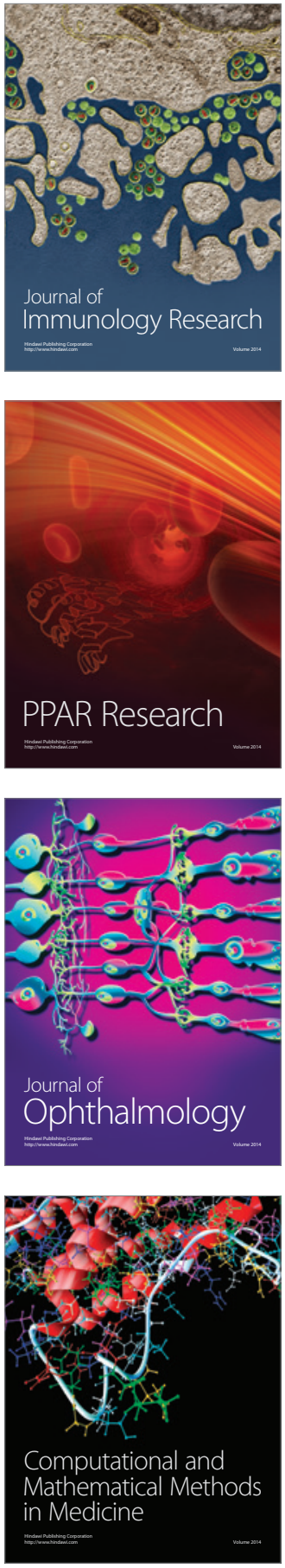

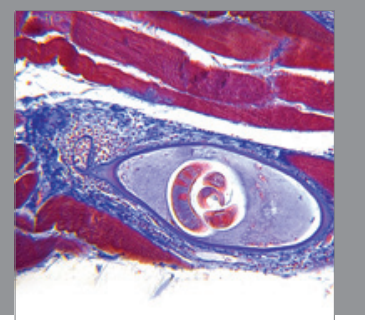

Gastroenterology

Research and Practice
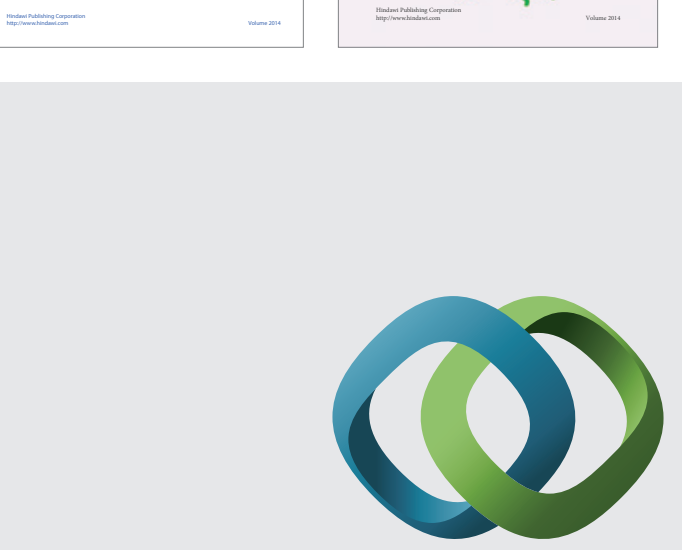

\section{Hindawi}

Submit your manuscripts at

http://www.hindawi.com
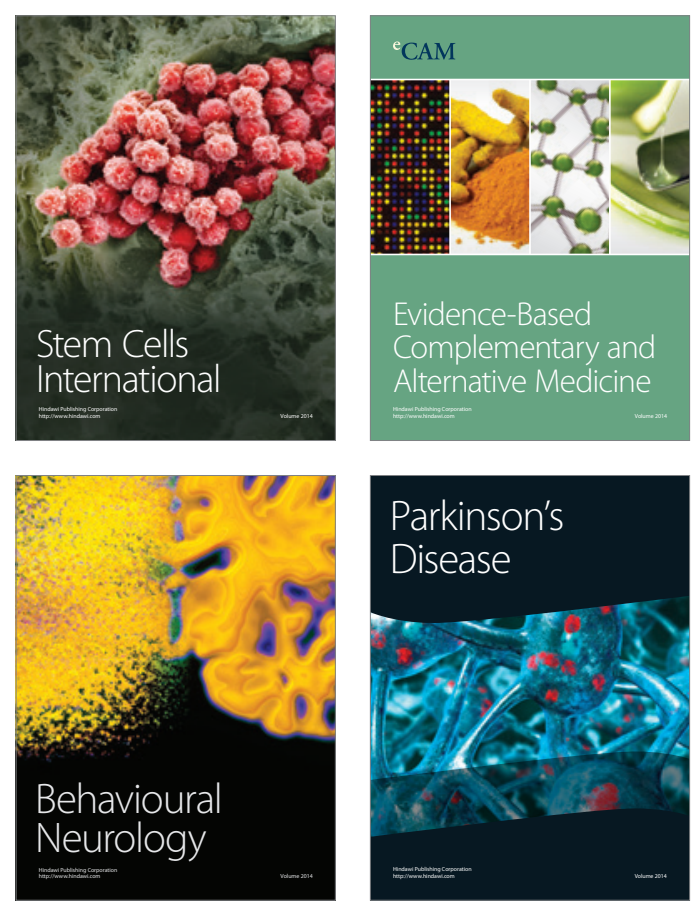

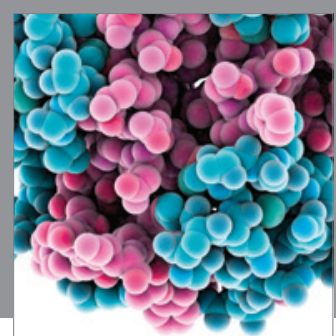

Journal of
Diabetes Research

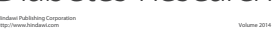

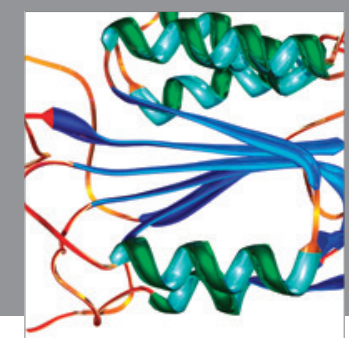

Disease Markers
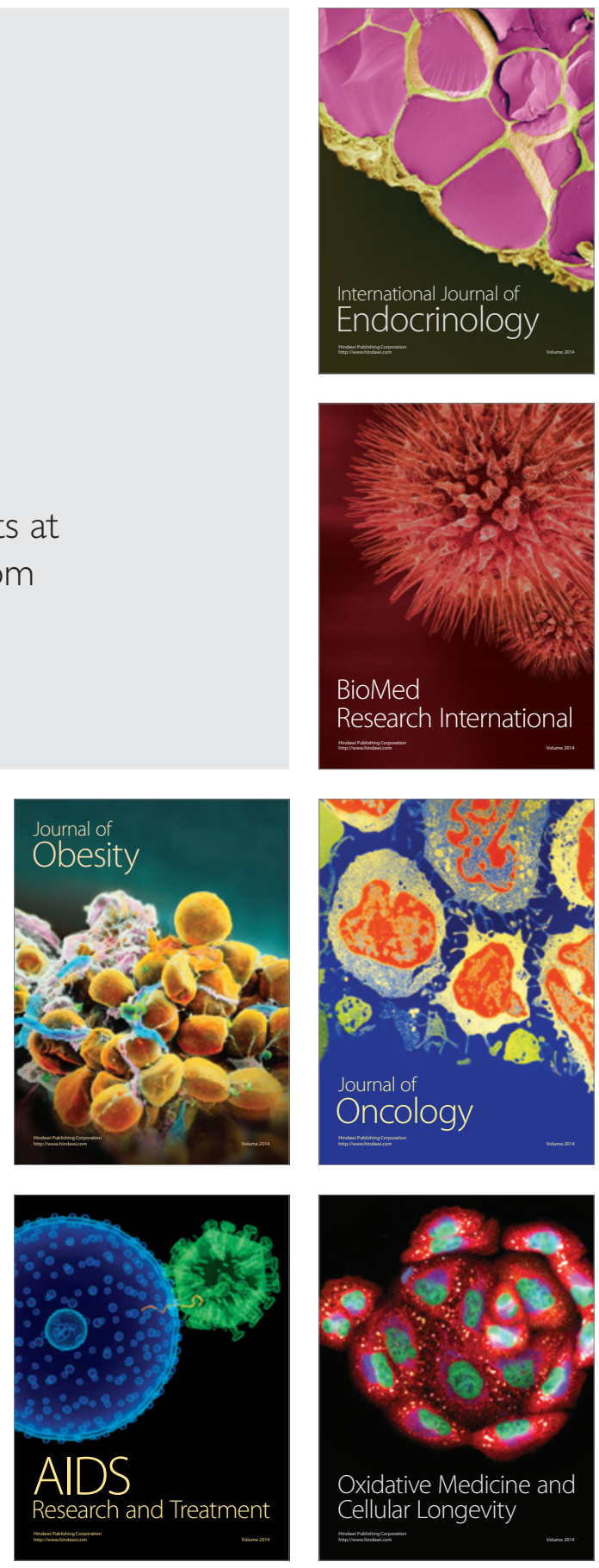